POS $\quad$ PROCEEDINGS

\title{
Proton Rich Nuclear Statistical Equilibrium
}

\author{
Ivo Seitenzahl* \\ University of Chicago and Max Planck Institute for Astrophysics \\ E-mail: irs@uchicago.edu
}

\section{F. X. Timmes}

Arizona State University

E-mail: fxt 44 @mac.com

\section{Abel Marin-Laflèche \\ École Polytechnique Palaiseau \\ E-mail: amarinlafleche@yahoo.fr}

\section{Edward Brown}

Michigan State University

E-mail: ebrown@pa.msu.edu

\section{Georgios Magkotsios}

University of Notre Dame

E-mail: gmagkots@nd.edu

\section{James Truran}

University of Chicago and Argonne National Labs

E-mail: truran@nova.uchicago.edu

Proton-rich material in a state of nuclear statistical equilibrium (NSE) is one of the least studied regimes of nucleosynthesis. One reason for this is that after hydrogen burning, stellar evolution proceeds at conditions of equal number of neutrons and protons or at a slight degree of neutron richness. Proton-rich nucleosynthesis in stars tends to occur only when hydrogen-rich material that accretes onto a white dwarf of neutron star explodes, or when neutrino interactions in the winds from a nascent proto-neutron star or collapsar-disk drive the matter proton rich prior to or during the nucleosynthesis. In this paper we solve the NSE equations for a range of proton-rich thermodynamic conditions. We show that cold proton-rich NSE is qualitatively different from neutron-rich NSE. Instead of being dominated by the iron-peak nuclei with the largest binding energy per nucleon that have a proton to nucleon ratio close to the prescribed electron fraction, NSE for proton-rich material near freeze-out temperature is mainly composed of ${ }^{56} \mathrm{Ni}$ and free protons. Previous results of nuclear reaction network calculations rely on this non-intuitive fact, which this paper will explain. We show how the differences and especially the large fraction of free protons arises as a direct result from the minimization of the Helmholtz free energy.

10th Symposium on Nuclei in the Cosmos

July 27 - August 1, 2008

Mackinac Island, Michigan, USA

\footnotetext{
* Speaker.
} 


\section{Introduction}

The nucleosynthetic production mechanism of some proton rich isotopes, in particular ${ }^{92,94} \mathrm{Mo}$ and ${ }^{96,98} \mathrm{Ru}$, is still uncertain. Recently, a new nucleosynthesis process, called the $v p$-process, was invented to explain the production of these isotopes [1]. In this process, nuclear matter ejected from the surface layers of a nascent proto neutron star is exposed to a strong neutrino flux which results in the following weak interactions:

$$
\begin{aligned}
& v_{e}+n \leftrightharpoons p+e^{-} \\
& \bar{v}_{e}+p \leftrightharpoons n+e^{+}
\end{aligned}
$$

Once the density is low enough that Pauli blocking is not a limiting factor, the mass difference between the neutron and the proton causes the neutrino interactions to be dominated by the forward reaction of eq. (1.1), which drives the nuclear matter proton rich. The matter then expands and assembles into NSE, which is not only proton-rich (i.e. $Y_{e}>0.5$ ) but actually contains a large mass fraction of free protons.

[2-6] also calculate nucleosynthesis in similar environments. The aforementioned proton rich isotopes (and other p-process nuclei) are then synthesized during the freeze out phase from the proton rich NSE state. The successful production of p-process nuclei crucially depends on the large number of free protons present at conditions when nuclear reactions fall out of equilibrium. The forward reaction of eq. (1.2) converts some of the free protons to neutrons, which allows nuclear matter to move past the "bottle neck" nucleus ${ }^{64} \mathrm{Ge}$ via the fast ${ }^{64} \mathrm{Ge}(n, p){ }^{64} \mathrm{Ga}$ reaction [1].

We show that the NSE mass fractions exhibit a great degree of symmetry across the line $Y_{e}=$ 0.5 for high temperatures where the composition is dominated by free nucleons and ${ }^{4} \mathrm{He}$. For colder temperatures, $\left(T_{9} \leq 6.0\right.$ and $\left.\rho \sim 10^{7} \mathrm{~g} \mathrm{~cm}^{-3}\right)$, the symmetry is broken and there are hardly any free neutrons present in the NSE state for $0.4<Y_{e} \leq 0.5$, whereas copious protons remain present for $Y_{e}>0.5$ at even colder temperatures. The successful production of $v p$-process nuclei crucially depends on this large abundance of free protons. It is well known from previous works that some free protons are present in the approach to NSE for $Y_{e} \lesssim 0.5$, [e.g. 7-10]. We show why this trend strongly amplifies for $Y_{e}>0.5$. Restricting ourselves to the nickel isotopic chain and free nucleons, we explain how the persistent large abundance of free protons in cold NSE arises as a result of a competition between the temperature dependent entropy term and the nuclear binding energy term contribution to the free energy.

At high temperature $\left(T_{9}=9.0\right)$ the NSE mass fractions are dominated by free nucleons and ${ }^{4} \mathrm{He}$. The ${ }^{4} \mathrm{He}$ mass fraction is symmetric across the line $Y_{e}=0.5$, and the mass fractions of free protons and neutrons are symmetric in a complementary sense - free protons are more abundant for $Y_{e}>0.5$ and free neutrons are more abundant for $Y_{e}<0.5$ (see fig. 1). At somewhat lower temperature $\left(T_{9}=6.5\right)$, the symmetry of the NSE mass fractions across the line of self-conjugacy is broken and only qualitatively discernible. For $\Delta Y_{e}>0$, free neutrons are less abundant for $Y_{e}=0.5-\Delta Y_{e}$ than are free protons for $Y_{e}=0.5+\Delta Y_{e}$ (see fig. 2). Furthermore, the mass fraction of ${ }^{4} \mathrm{He}$ is not symmetric anymore and the abundance peaks of the iron peak nuclei are wider on the proton rich side. At even lower temperature $\left(T_{9}=3.5\right)$, the qualitative features of the mass fractions of nuclei in NSE as a function of $Y_{e}$ at fixed density and temperature change dramatically in the transition from the neutron rich to the proton rich side (see fig. 3). For $Y_{e}>0.5$ the mass 


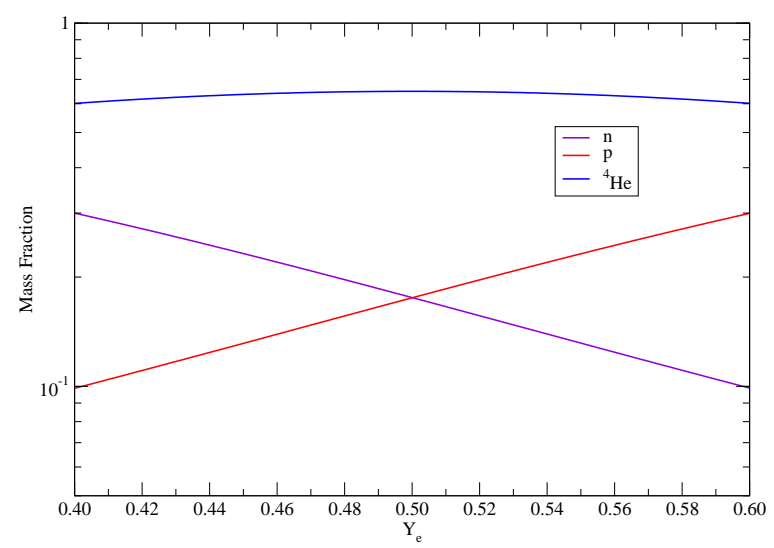

Figure 1: Mass fractions of nuclei in NSE as a function of electron fraction for a constant density $\rho=$ $10^{7} \mathrm{~g} \mathrm{~cm}^{-3}\left(n_{B}=6.0 \times 10^{30} \mathrm{~cm}^{-3}\right)$ and relatively high temperature $T=9.0 \times 10^{9} \mathrm{~K}$. Shown are all nuclei with mass fractions larger than $10^{-2}$. The mass fractions exhibit large degree of (in the case of nucleons complementary) symmetry across $Y_{e}=0.5$.

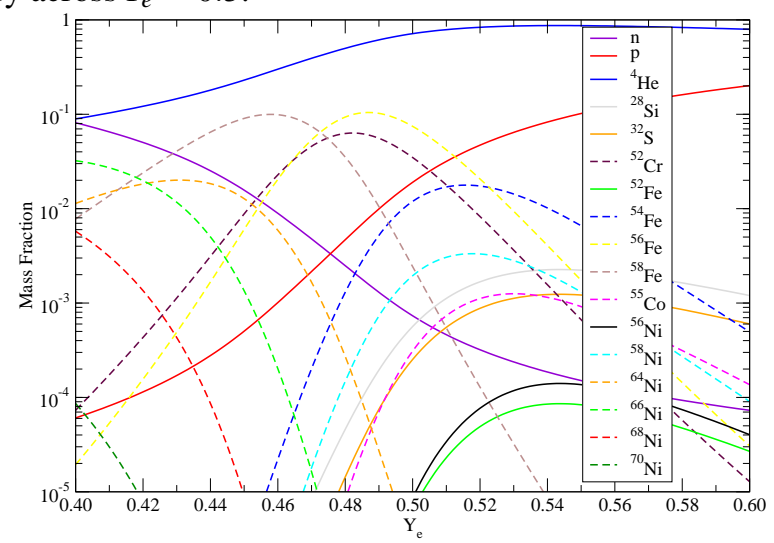

Figure 2: Mass fractions of nuclei in NSE as a function of electron fraction for a constant density $\rho=10^{7} \mathrm{~g}$ $\mathrm{cm}^{-3}\left(n_{B}=6.0 \times 10^{30} \mathrm{~cm}^{-3}\right)$ and temperature $T=6.5 \times 10^{9} \mathrm{~K}$. Shown are some abundant nuclei with mass fractions larger than $10^{-5}$. The symmetry across $Y_{e}=0.5$ is already broken.

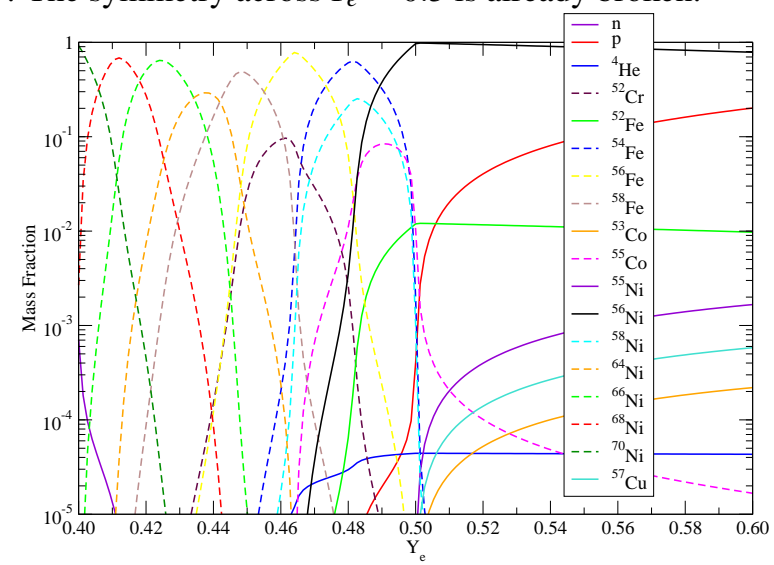

Figure 3: Mass fractions of nuclei in NSE as a function of electron fraction for a constant density $\rho=10^{7} \mathrm{~g}$ $\mathrm{cm}^{-3}\left(n_{B}=6.0 \times 10^{30} \mathrm{~cm}^{-3}\right)$ and temperature $T=3.5 \times 10^{9} \mathrm{~K}$. Shown are some abundant nuclei with mass fractions larger than $10^{-5}$. The mass fractions on either side of $Y_{e}=0.5$ exhibit qualitatively very different behavior. 


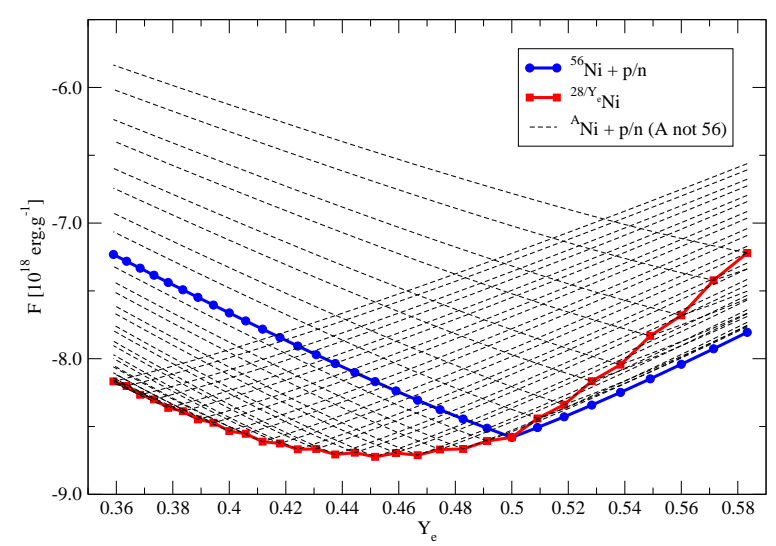

Figure 4: Helmholtz free energy of compositions consisting of nickel isotopes and nucleons as a function of $Y_{e} . T=3.5 \times 10^{9} \mathrm{~K}$. and $n_{B}=6.0 \times 10^{30} \mathrm{~cm}^{-3}$. For $Y_{e}>0.5$, a mix of protons and test isotope ${ }^{56} \mathrm{Ni}$ (thick blue line with dots) is favored. Other test isotopes (dotted lines) result in larger $\mathscr{F}$. For $Y_{e}<0.5$, a pure isotope composition (thick red line with squares) yields the lowest free energy.

fraction distribution of the iron peak nuclei is no longer a sequence of peaks; instead ${ }^{56} \mathrm{Ni}$ remains the most abundant nuclear species by mass all the way out past $Y_{e}=0.60$ while the mass fraction of free protons continues to rise.

\section{Discussion}

In NSE, the Helmholtz free energy $\mathscr{F}=(U-Q)-T S$ is minimized with respect to the nuclide mass fractions [e.g. 11,12]. We compare the free energy of compositions at fixed $Y_{e}$ and constant baryon density $n_{B}$ and temperature $T$, one consisting of pure composition of a reference nucleus from the nickel isotopic chain, and the others consisting of a mix of free nucleons (either protons or neutrons to give the same $Y_{e}$ ) and a "test" nickel nucleus. Using the data from the latest atomic mass evaluation and an equation of state we compute and compare $\mathscr{F}$ for such compositions for the whole $\mathrm{Ni}$ isotopic chain, with each isotope as the test nucleus at a time. The free energies of all such compositions are shown as a function of $Y_{e}$ in fig. 4. It clearly shows that for $Y_{e}<0.5$ a pure composition of the nickel isotope with $Y_{e}=28 / A$ has the lowest free energy, and that for $Y_{e}>0.5$ a composition consisting of free protons and ${ }^{56} \mathrm{Ni}$ minimizes $\mathscr{F}$. Other choices for the test nucleus have higher free energy (dotted lines in fig. 4).

\section{Conclusions}

We have presented mass fraction trends for NSE in proton rich environments. We have explained the high free particle (proton) mass fractions for $Y_{e}>0.5$ and large mass fraction of ${ }^{56} \mathrm{Ni}$ even out to $Y_{e}=0.6$ by considering a simplified model. Restricting ourselves to the nickel isotopic chain, we have explicitly shown that the free energy for $Y_{e}>0.5$ is minimized by a state consisting of a mixture of free protons and the self-conjugate ${ }^{56} \mathrm{Ni}$, whereas for $Y_{e}<0.5$ a state with a pure composition made up of the nickel isotope that has $\mathrm{Z} / \mathrm{A}$ closest to the prescribed $Y_{e}$ is preferred. 
In reality, other isotopic chains are of course accessible. Other iron peak nuclides (especially the slightly neutron rich even isotopes of iron) compete with nickel isotopes for the most tightly bound nucleus with Z/A near $Y_{e}$ (see fig. 3), giving the familiar NSE abundance pattern.

\section{Acknowledgments}

This work is supported at the University of Chicago by the Department of Energy under Grant B523820 to the ASC/Alliances Center for Astrophysical Thermonuclear Flashes, and the National Science Foundation under Grant PHY 02-16783 for the Frontier Center "Joint Institute for Nuclear Astrophysics" (JINA), and at Argonne National Laboratory by the U.S. Department of Energy, Office of Nuclear Physics, under contract DE-AC02-06CH11357. EFB is supported by the NSF under grant AST 05-07456.

\section{References}

[1] Fröhlich, C., Martínez-Pinedo, G., Liebendörfer, M., Thielemann, F.-K., Bravo, E., Hix, W. R., Langanke, K., \& Zinner, N. T. 2006, Physical Review Letters, 96, 142502

[2] Meyer, B. S. 1994, ARAA, 32, 153

[3] Jordan, IV, G. C. \& Meyer, B. S. 2004, ApJL, 617, L131

[4] Pruet, J., Woosley, S. E., Buras, R., Janka, H.-T., \& Hoffman, R. D. 2005, ApJ, 623, 325

[5] Pruet, J., Hoffman, R. D., Woosley, S. E., Janka, H.-T., \& Buras, R. 2006, ApJ, 644, 1028

[6] Wanajo, S. 2006, ApJ, 647, 1323

[7] Truran, J. W., Cameron, A. G. W., \& Gilbert, A. 1966, Canadian Journal of Physics, 44, 563

[8] Bodansky, D., Clayton, D. D., \& Fowler, W. A. 1968, ApJS, 16, 299

[9] Hix, W. R. \& Thielemann, F. K. 1996, ApJ, 460, 869

[10] The, L.-S., Clayton, D. D., Jin, L., \& Meyer, B. S. 1998, ApJ, 504, 500

[11] Nadyozhin, D. K. \& Yudin, A. V. 2004, Astronomy Letters, 31, 271

[12] Seitenzahl, I. R., Townsley, D. M., Peng, F., \& Truran, J. T. 2008, Atomic Data and Nuclear Data Tables, in press 